\title{
Exploring the Impact of Hurricane Maria on Parents and Health Care Personnel of a Neonatal Intensive Care Unit in Puerto Rico
}

\author{
Liza C. Sánchez-Plazas, MD, FAAP; Inés García-García, MD, FAAP; \\ Lourdes García-Fragoso, MD, FAAP
}

$\mathrm{H}$ aving a baby hospitalized in the neonatal intensive care unit (NICU) is a very stressful situation for parents, but when this occurs concomitantly with a natural disaster, the stress can become magnified. Studies revealed that parents with an infant in the NICU have a higher rate of depression, anxiety, stress, loss of control, and trauma symptoms. ${ }^{1,2}$

On September 20, 2017, Puerto Rico received the impact of catastrophic Category 4 Hurricane Maria. The unpreparedness of the island severely affected basic services and health care. There is scant published literature about the impact of a major natural disaster affecting the NICU and its consequences. In the United States, 2 major hurricanes, Katrina and Sandy, affected the states of Louisiana and New York respectively, with catastrophic effects. Those studies centered on the experience of an emergent evacuation of their NICU and lessons for preparedness in case of another natural disaster. ${ }^{3,4}$ This is vital because we should take into account the possible separation of the infants from their family during a natural disaster.

We retrospectively analyzed the experiences of parents and health care providers of our NICU during Hurricane Maria through a cross-sectional study. Questionnaires were given to parents to assess social needs and symptoms of acute stress post-hurricane and to health care workers to document what they endured. Thirty-four parents (66\% females) answered the questionnaire. Parents' biggest fears were "NICU windows would break" (94\%) and "baby's supporting machines not working" (88\%). Median days until parents were able to visit were 4 days (1-9 days). Parents reported sleeping problems (30\%), lack of concentration (24\%), feeling tense (21\%), and feeling helpless $(26 \%)$. Eighty health care providers ( $81 \%$ females) completed the questionnaire. About $40 \%$ stayed in the hospital during the storm, some (44\%) stayed for at least 2 days, and some (3\%) remained as long as a week without seeing their families; $9 \%$ lost their homes.

Hurricane Maria exposed our weaknesses and lack of preparedness in the event of a major disaster. Preparedness plans should be elaborated with anticipation, even during the admission process, because of our susceptibility to hurricanes based on our geographical location. They should include how to support parents in this critical period where their ability to cope and adequately manage the situation may be disrupted. Because parents are not allowed to stay in the unit during an emergency, the communication with families' prior, during, and after a disaster is vital to help reduce their fears about their infants' safety.

Additionally, we must review our preparedness plan as to how to provide adequate support to health care providers. They are also separated from their families while providing care in the NICU and may be significantly impacted by the natural disaster. Even without knowing how their family members fared after the storm, they continued working and striving to provide optimal care to NICU patients while trying to hide their concerns, fears, and fatigue. Emotional support to health care workers and responders is also crucial to prevent crisis and reduce psychosocial impact in this population.

\section{About the Authors}

University of Puerto Rico School of Medicine, Department of Pediatrics, Neonatology Section, San Juan, PR (Drs Sánchez-Plazas, GarcíaGarcía, García-Fragoso)

Correspondence and reprint requests to Lourdes García-Fragoso, UPR School of Medicine, Department of Pediatrics, Neonatology Section, PO Box 365067, San Juan, PR 00936-5067 (e-mail: lourdes. garcia1@upr.edu).

\section{Conflict of Interest Statement}

The authors have no conflicts of interest to declare. 


\section{REFERENCES}

1. Obeidat $\mathrm{H}$, Bond $\mathrm{E}$, Callister $\mathrm{L}$. The parental experience of having an infant in the newborn intensive care unit. J Perinat Educ. 2009;18(3):23-29.

2. Cox S, Allen A, Winter I. Measuring neonatal intensive care unit-related parental stress. J Adv Nurs. 2005;49(6):608-615.
3. Barfield WD, Krug SE. Committee on fetus and newborn, AAP Disaster Preparedness Advisory Council. Disaster preparedness in neonatal intensive care units. Pediatrics. 2017;139(5):e1-e11.

4. Espiritu M, Patil U, Cruz $\mathrm{H}$, et al. Evacuation of a neonatal intensive care unit in a disaster: lessons from Hurricane Sandy. Pediatrics. 2014;134(6): e1662-e1668. 